\title{
Modelling Psychopathology Development in Psychiatric Illnesses Using Cognitive Schema Theory and Genetic-Environmental Interactions
}

\author{
Irosh Fernando, Member, IACSIT
}

\begin{abstract}
A deeper understanding of the process of psychopathology development is invaluable not only for taking the necessary preventive measures against the development of psychiatric disorders, but also to predict the course of a psychiatric illnessin a given individual. Even though genetic and environmental influences are implicated in this process, and there are number of psychological theories that explain psychopathology, the exact mechanism of this process is unknown. This paper attempts to explain this mechanism using a mathematical model based on Cognitive Schema Theory and genetic-environmental interactions.
\end{abstract}

Index Terms-Mathematical modelling of psychopathology development, mathematical modelling of cognitive schema theory, mathematical models in psychology and psychiatry, modelling genetic-environmental interactions.

\section{INTRODUCTION}

Similar to those rather objective pathological phenomena (e.g. tumours, hormonal deficits) observed in physical illnesses, there are pathological phenomena (e.g. persecutory delusions, nihilistic thoughts, low self-esteem) known as psychopathology observed in psychiatric illnesses. Because these phenomena are highly subjective, and involves individual's psychological experiences, it has been difficult to study the process of their development that leads to psychiatric disorders. As a result, aetiologies of most of the psychiatric disorders are poorly understood compared to physical illnesses. For example, whilst the aetiology of an infectious disease such as meningitis is well understood, the aetiology of a psychiatric illness such as depression is a relative mystery. Even though both genetic and environmental factors are generally implicated in its aetiologyin a rather superficial way, how exactly this interaction leads to development of psychopathology is not known. Whilst psychiatric disorders have been recognized as some of the most disabling illnesses [1], development of effective preventive( e.g. vaccinations for infectious diseases) and treatment strategies have been extremely challenging due to lack of deeper understanding of their aetiological processes.

This paper attempts to take a step forward in describing such processes by introducing a mathematical model that describes the process of psychopathology development, and

Manuscript received March 12, 2013; revised May 20, 2013.

Irosh Fernando is with the School of Electrical Engineering and Computer Science, University of Newcastle and the Calvary Mater Hospital, Hunter New England Local Area Health District, NSW, Australia (e-mail: Irosh.fernando@uon.edu.au, Irosh.fernando@hnehealth.nsw.gov.au). the observed characteristics of this process. The introduced model is based on current understanding of genetic and environmental interactions and also the Cognitive Schema Theory [2], which is one of the elaborated theoretical frameworks that explain psychopathology from a psychological perspective. The author believes that mathematical modelling of psychological processes is important not only for achieving a deeper understanding of these, but also to develop necessary preventive measures against psychiatric disorders, and make predictions about individual's psychological development.

\section{RELATEd PREVIOUS APPROACHES}

Unfortunately, mathematical approaches to understand the aetiological processes of psychiatric disorders are lacking in the literature. One of the possible reasons for this situation is the difficulty of representing highly subjective phenomena as numerical variables. However, at least there are two somewhat related approaches, even though, as described below, their aims are considerably different from the focus of this paper.

There has been a previous attempt to model the gene environmental interaction using the following probability function [3], which gives the probability of liability to a psychiatric illness, $X$ exceeding a threshold, $t$.

$$
P_{i j}(X>t)=\int_{t}^{\infty} \emptyset_{i j}(X) d X
$$

In the above model, $\emptyset_{i j}(X)$ is a probability density function, which computes the probability that an individual with genotype, $i$ and the measured environment $j$ is affected by a psychiatric illness. The main aim of this study has been to explain possible artifacts of the claims on genetic-environmental interactions that have been reported by previous studies. Therefore, this model is not able to properly explain the influence of genetic and environmental interactions on psychopathology development described in the next section of this paper.

Also, a mathematical model, known as a general quantitative theory has been proposed for explaining the process of personality development [4]. The character traits described in this theory provides an alternative perspective on personality compared to the Cognitive Schema Theory in this paper. The rate of change of a given character trait, $x$ with time, $t$ and its fitness (i.e. how adaptive the behavior determined bythe character trait, $x$ is) is modeled by the following differential equation. 


$$
\frac{d x(t)}{d t}=\frac{d W(x)}{d x}+\varepsilon
$$

where $\varepsilon$ is a random variable.

However, the Cognitive Schema Theory provides a relatively rich framework for understanding psychopathology. Also, the general quantitative theory mainly focuses on personality development rather than psychopathology. Therefore, it is not able to describe the known characteristics of genetic and environmental interactions on psychopathology development described in the following section.

\section{GENETIC AND ENVIRONMENTAL INFLUENCE ON PSYCHOPATHOLOGY DEVELOPMENT}

There is a compelling body of research evidence indicating both genetic and environmental influences on the development of psychopathology resulting in psychiatric disorders [5]-[8]. For example, it is known that those who are exposed to adverse environmental conditions (e.g. abuse and trauma) as well as those who have biological parents with psychiatric disorders are vulnerable to develop psychiatric illnesses. In certain psychiatric disorders such as depression, specific abnormalities of certain genes such as Serotonin transport gene are implicated [9]. However, not everyone who has environmental influence, or genetic influence, or even both goes onto develop psychopathology and a psychiatric illness. An important characteristic of the genetic-environmental influence is that those who have a higher genetic influence tend to be more affected compared to those with low genetic influence, under conditions where environmental influence is held as a constant. For example, one of the seminal studies in genetic and environmental interactions related to traits of disruptive behavior, has revealed that individuals with a high genetic influence tend to have a higher impact of environmental influence on criminality compared to those with a low genetic influence [10].

Any given psychopathology has a state of severity that tends to fluctuate over the course of time. For example, paranoia is a psychopathology, which may manifest as a person having difficulties with trusting people at a state of low severity, and as it increases in severity it transforms into persecutory thoughts, over-valued ideas, and then persecutory delusions resulting in a psychotic illness. Exacerbation of the severity of a psychopathology is often followed by an exposure to adverse environmental stimuli such as severe stress often for a prolonged period of time. As the exposure to an adverse environmental situation is removed, severity of psychopathology abates leading to a recovery, or even aversion from the development of further psychiatric illness. When the environmental conditions remain relatively unchanged, severity of a psychopathology may reach a stable state. These states could be either healing, partial recovery, or continuation of illness in its full-blown state depending on the severity of environmental influence (i.e. adversity).

\section{UNDERSTANDING PSYCHOPATHOLOGY USING COGNITIVE SCHEMA THEORY}

Genetic and environmental influence described above is a general process, and only provides a partial understanding of psychopathology development. For example, it is not able to explain the formation of different types of psychopathology. In order to achieve a better understanding of this process, an appropriate theory that describes human psychopathology has to be used. In Psychiatry, there are many such theories. For example, Self-psychology [11], Object-relations Theory [12], Ego-psychology [13], Cognitive Schema Theory, and Attachment Theory [14]-[16]. Compared to other theories, the concepts described in Cognitive Schema Theory are relatively easy to model mathematically, and therefore, this theory was chosen to complement the understanding provided by genetic and environmental influences on psychopathology development. According to Cognitive Schema Theory, psychopathology can be understood in relation to eighteen different cognitive schemas. For example, paranoia, and low self-esteem can be understood in relation to cognitive schemas of mistrust and defectiveness respectively. Individuals use cognitive schemas to interpret their experiences with the environment. State of each cognitive schema is dynamic, and determined by both genetic and environmental influence. The state of any given cognitive schema can shift towards an extreme of over-activation resulting in psychopathology and psychiatric illnesses, especially when the person experiences prolonged adverse environmental influences. Similarly, it can shift towards an extreme of under-activation under healthy environmental influence, thus averting the risk of developing psychopathology. For example, in relation to the cognitive schema of mistrust, if a vulnerable individual is exposed to a situation in which he/she is mistreated, exploited and abused for a prolonged period of time, over-activation of the cognitive schema may result in the development of persecutory thoughts, and then even persecutory delusions in a vulnerable individual. On the other hand, if the same individual is alternatively exposed to a relatively benign environment in which he/she is treated generously by others, and his/her trust is not breached, the cognitive schema starts to become underactive leading to healing and recovery over a prolonged period of time. Development of a psychopathology can be conceptualized as over activation of its related cognitive schema beyond a critical point as described in Fig. 1.

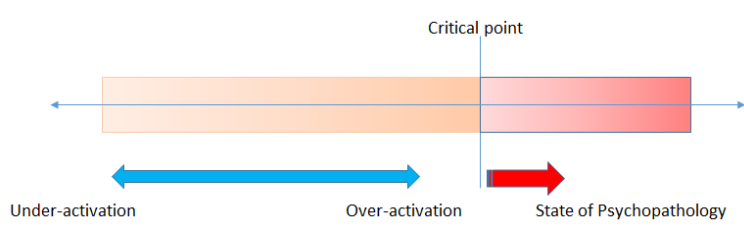

Fig. 1. Extremes of cognitive schema states and development of psychopathology.

\section{MODEL FORMULATION}

Cognitive Schemas can be represented as a vector, $C=\left(c_{1}, \ldots, c_{n}\right)$, whereas all states of these schemas can be represented as a corresponding vector, $S=\left(s\left(c_{1}\right), \ldots, s\left(c_{n}\right)\right)$, where, $s$ is a function defined as follows.

$s: C \rightarrow\left[s_{L} \ldots s_{U}\right], s_{L}, s_{U} \in \mathbb{R}$, where $s_{L}$ and $s_{U}$ are the lower and upper ends of the range, in which the state of activation (i.e. severity) of cognitive schema fluctuates. $s_{U}$ 
represents the critical point described in Fig. 1.

Similarly, let us define the environmental influence as a quantitative variable, $r \in\left[r_{L} \ldots r_{U}\right], r_{L}, r_{U} \in \mathbb{R}$, where $r_{L}$ and $r_{U}$ are the lower and upper ends of the range of values of environmental influence (i.e. best possible and worst possible environmental influences respectively).

Next, we define the genetic influence also as a quantitative variable, $g \in\left[g_{L} \ldots g_{U}\right], g_{L}, g_{U} \in \mathbb{R}$, where $g_{L}$ and $g_{U}$ are the lower and upper ends of the range of values of genetic influence(i.e. lowest and highest genetic influence respectively).

For convenience we simplify the notation, $s\left(c_{i}\right)$ of $i$ th cognitive schema, to $S$ and we define, $S_{i}: i=0 \ldots n$ as the state of any given cognitive schema at $i$ th unit of time.

Progressive change of the state of any given cognitive schema, under an environmental influence, $r$ in an individual with a genetic influence, $g$ can be modeled as follows.

$$
S_{n+1}=r S_{n}+g
$$

Given that there is only genetic influence at the starting point of life, let us define $S_{o}=g$. Now, the model can be described as a dynamic system of equations that represent the evolution of the state of any given cognitive schema as he/ she undergoes various environmental experiences starting from birth.

$$
\begin{gathered}
S_{o}=g \\
S_{1}=r S_{0}+g \\
S_{2}=r S_{1}+g \\
S_{n+1}=r S_{n}+g
\end{gathered}
$$

Also,

$$
\begin{gathered}
S_{1}=r S_{0}+g=r g+g=g(r+1) \\
S_{2}=r S_{1}+g=r g(r+1)+g=g\left(r^{2}+r+1\right) \\
S_{3}=r S_{2}+g=g\left(r^{2}+r+1\right)+g=g\left(r^{3}+r^{2}+1\right) \\
S_{n}=g\left(r^{n}+r^{n-1}+\cdots+1\right) \\
S_{n}=g \sum_{i=0}^{n} r^{i}
\end{gathered}
$$

Given that $S_{n+1}=r S_{n}+g$, if the series given in equation (2) converges,

$$
S_{n+1}=S_{n}
$$

By substituting the above to equation (1),

$$
\begin{gathered}
S_{n}=r S_{n}+g \\
S_{n}-r S_{n}=g \\
S_{n}(1-r)=g \\
S_{n}=\frac{g}{1-r}, r \neq 1
\end{gathered}
$$

Given that $S_{n}$ is restricted to the interval, $\left[s_{L} \ldots s_{U}\right]$, and $\operatorname{Max}\left(S_{n}\right)=s_{U}$, then $g<s_{U}(1-r)$ should hold in order to achieve the convergence within this interval. Situations, in which $S_{n}$ does not converge within the above interval, can be interpreted clinically as the development of psychiatric illness. Also, a threshold function, $f\left(s_{n}\right)$ with a value, $t \in\left[s_{L} \ldots s_{U}\right]$ can be used define a cut-off point for grading cognitive schema status for various clinical purposes. For example, to decide if an early therapeutic intervention is necessary in order to prevent development of a psychiatric illness, $f\left(s_{n}\right)$ can be defined as follows.

$$
f\left(s_{n}\right)= \begin{cases}\text { Therapy required }=\text { false, } & s_{n}<t \\ \text { Therapy required }=\text { true, } & s_{n} \geq t\end{cases}
$$

Given the fact that the variable $g$ is a constant, the only control an individual can exert on the process of psychopathology development is to change his/her environment by changing $r$ in order to achieve an expected outcome (i.e. aversion of the development of psychopathology or healing).

\section{Model AsSUMPTIONS AND RESTRICTIONS}

The proposed model only explains the evolution of cognitive schema states into psychopathology resulting in psychiatric illnesses (i.e. over-activation reaching the critical point described in Fig. 1). It is important to note that the model does not explain recovery once psychopathology is established. Recovery from an established psychopathology, is a slow process, and involves biological parameters (e.g. changes in neurotransmitters and their receptors), which are not included in the current model. For example, delusions and hallucinations will not improve merely by changing the environment, but also require antipsychotic medications in order to correct the defective functioning of neurotransmitters and their receptors. The model is also based on the following assumptions.

\section{A. Environmental Influence}

Often an individual's environment remains stable for discrete time intervals (e.g. key relationships, workplace, and place of residence). Therefore, the model assume that environmental influence remains constant for a given period of time. Environment influence can also be interpreted as an approximation of individual's experience as an average for a given time period.

\section{B. Absence of Interactions between Different Cognitive Schemas}

The model assumes that there are no interactions between the different cognitive schemas (i.e. change of the status of one cognitive schema does not influence the change of another).

\section{Linear Process}

The model assumes that the process of psychopathology development via genetic-environmental interactions resulting in changes in cognitive schema states follows a linear process.

\section{SiMULATION OF THE MODEL}

Simulation of the model is important in order to observe if the model behaves in a similar way to known real world situations. This section describes the simulation results of 
several commonly observed general clinical patterns. Simulation results discussed below are valid for any given cognitive schema, and do not refer to any specific cognitive schema or psychopathology.

The proposed model was programmed in MATLAB, and the model parameters were initialized as follows.

$$
\begin{array}{cc}
s_{L}=0, & s_{U}=1 \\
r_{L}=0, & r_{U}=1 \\
g_{L}=0, & g_{U}=0.5
\end{array}
$$

Individuals are not born with psychopathologies such as persecutory delusions and low self-esteem. Therefore, $g_{U}$ should be less than $s_{U}$, and a reasonable subjective approximation would be $g_{U}=0.5$ as stated above.

\section{A. Different Genetic Influences, but Same Environmental Influence}

Let us simulate psychopathology development processes of three different individuals in relation to a single cognitive schema.Assume that the all three individuals has the same environment influence, $r=0.5$, and their genetic influences are given in the following vector.

$$
\boldsymbol{g}=\left(\begin{array}{l}
0.1 \\
0.3 \\
0.5
\end{array}\right)
$$

As described in Fig. 2, the same cognitive schema in each individual reaches different steady states in the same order of their genetic influences. As expected, the individual with the highest genetic influence achieves the highest schema state compared to other individuals.

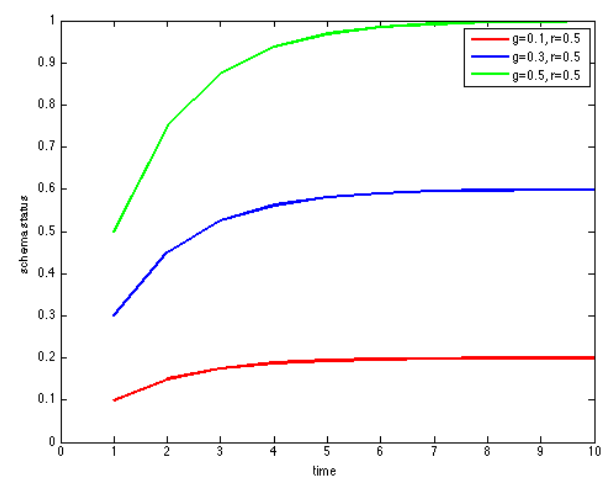

Fig. 2. Trajectories of cognitive schema status in three individuals under the same environment influence.

\section{B. Different Environmental Influences, but Same Genetic Influence}

Now, let us simulate the psychopathology development processes of three different individuals with an equal level of genetic influence, $g=0.4$, but each having different environment influences (e.g. monozygotic twins adopted by three families), in relation to a given cognitive schema. Their environment influences are given in the following vector.

$$
\boldsymbol{r}=\left(\begin{array}{l}
0.2 \\
0.5 \\
0.8
\end{array}\right)
$$

The results are described in Fig. 3. As expected, cognitive schema in each individual reaches different states in the same order of their environmental influences, and the individual with highest environmental influence develops psychopathology resulting in a psychiatric illness.

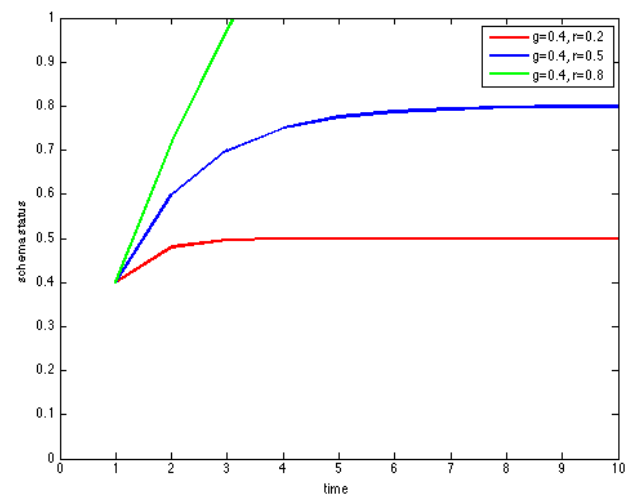

Fig. 3. Trajectories of cognitive schema status in a three individuals with equal genetic influences under three different environment influences.

\section{Environmental Influence and Genetic Influence Are Swapped}

Now, let us consider two individuals with genetic and environmental influences given in the following vectors.

$$
\boldsymbol{g}=\left(\begin{array}{l}
0.2 \\
0.5
\end{array}\right), \boldsymbol{r}=\left(\begin{array}{l}
0.5 \\
0.2
\end{array}\right)
$$

The values of genetic and environmental influence in the first individual has been swapped in the second individual. As expected, genetic influence dominates environmental influence (Fig. 4).

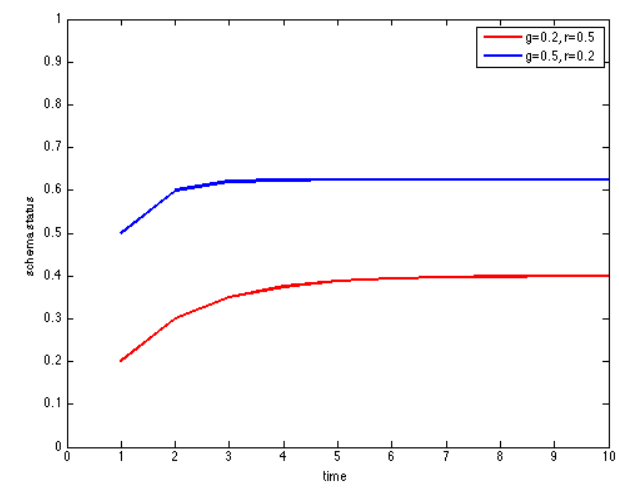

Fig. 4. Trajectories of cognitive schema states in two individuals with their values for genetic and environmental influences swapped.

\section{Early Interventions and Preventions of Psychiatric Illnesses}

Now, suppose there is an individual with a moderate genetic influence, $g=0.3$ continues to be under an adverse environmental influence, $r=0.8$. If the environment is changed to a more benign one with $r=0.2$, the trajectory of psychopathology development is averted as described in Fig. 5.

When an individual is at risk of developinga psychopathology, early treatment interventions involve modifying his/her environmental influence. Some early intervention programs may temporarily remove the individual from an adverse environment, and once this environmental influence is sufficiently improved, the 
individual is returned to his/her original environment (e.g. government interventions to remove children who are at risk from their parents). Often the modified original environmentmay not be as healthy as the temporary environment (e.g. rehabilitation facility). Therefore even though the risk of developing a psychopathology is averted, the cognitive schema state may still reach a higher level compared to what it would have reached if the individual continued to remain in the original environment. This scenario can be modeled by changing the environmental influence to an intermediate value, $r=0.6$ in the individual described above. The results are described in Fig. 6.

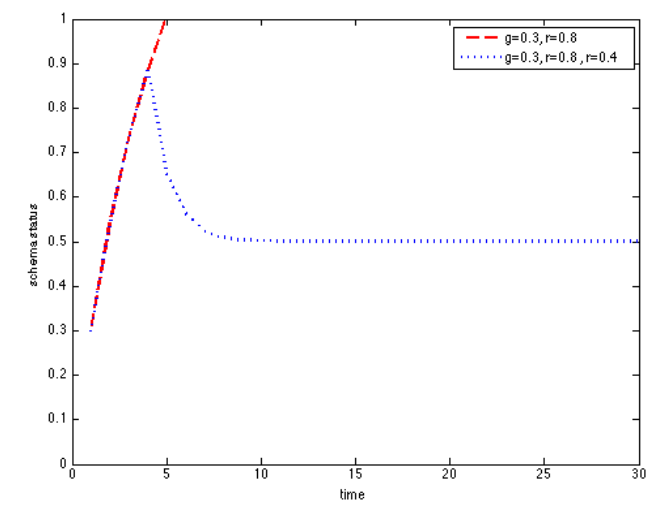

Fig. 5. Trajectories of cognitive schema states after changing environmental influence.

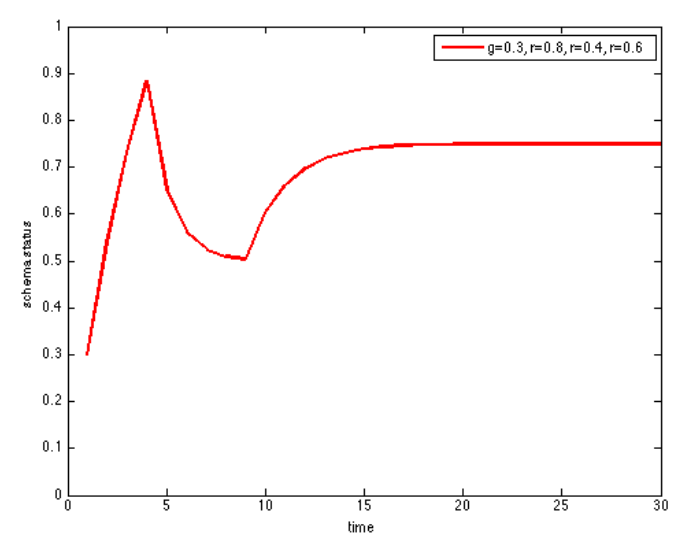

Fig. 6. Trajectories of cognitive schema states after changing environmental influence twice.

\section{E. Different Environmental Influences at Different Time Periods}

In reality, people's environmental situations changes (e.g. study, employment, family, relationships). As a result, the environmental influence changes in the same individual at different times. In order to model this situation, let us consider two individuals with different genetic influences having three different environmental influences during three consecutive time periods, as given in following vectors.

$$
\boldsymbol{r}=\left(\begin{array}{l}
0.2 \\
0.5 \\
0.3
\end{array}\right), \boldsymbol{g}=\left(\begin{array}{l}
0.2 \\
0.4
\end{array}\right)
$$

As expected the impact of environmental influence is greater on the individual with higher genetic influence $(g=0.4)$. Nonetheless, as described in Fig. 7 , it does not lead to a psychiatric illness. However, suppose the genetic influence is higher $(g=0.5)$. In this situation, as described in Fig. 8, under environment influence, $r=0.5$, cognitive schema state rapidly increases, but doesn't converge within the prescribed interval, resulting in a psychiatric illness. This explains why only genetically vulnerable individuals develop psychiatric illnesses, while less genetically vulnerable individuals, under the same environmental influence, are able to live without developing such psychopathologies.

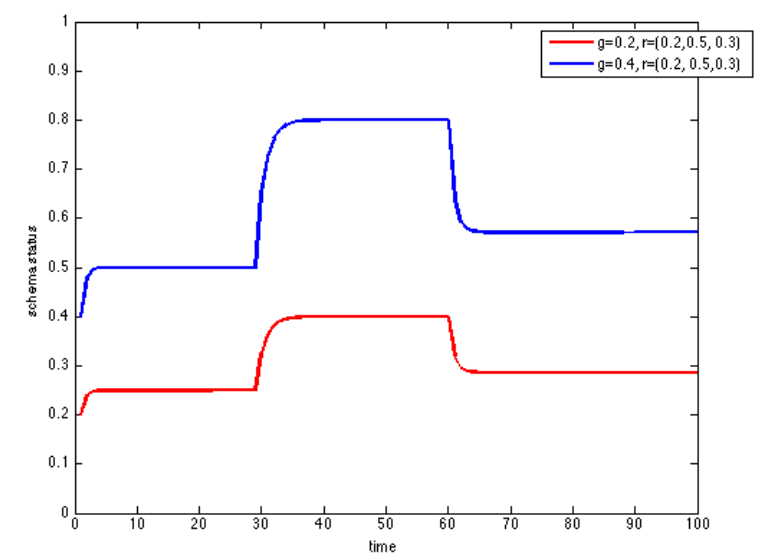

Fig. 7. Trajectories of cognitive schema states under three different environmental influences.

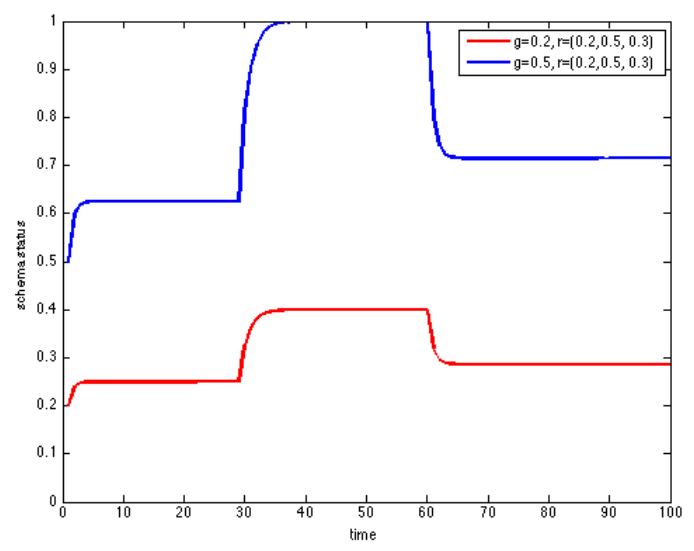

Fig. 8. Development of psychopathology in a genetically vulnerable individual.

\section{DISCUSSION}

The proposed model is able to describe the mechanism of the observed characteristics of psychopathology development process under genetic-environmental influence. These include: increased vulnerability of those who have a high genetic predisposition; evolution of cognitive schema states into psychopathology under prolonged adverse environmental influences; aversion from psychiatric illness and healing when a vulnerable individual is removed from an adverse environmental influence, and placed in a healthy environment. The model enables us to answer useful clinical questions, such as "For a given genetic influence, what should be the minimum environmental influence required in order to prevent development of a psychopathology ?", and " How long it will take for a vulnerable individual with a known genetic influence, placed under a measured environmental influence, to develop a psychopathology?"

One of the challenges the proposed model faces is the question 'How do we represent the genetic and environmental influences numerically in a meaningful and 
consistent way?' With the advancement of genetics and the area of biomedical signal processing, it is possible to derive estimates for the parameters of the model and this will be an important area of further work. On the other hand, expert clinicians can use their subjective judgment to estimate the parameters and use the model to study clinical scenarios of psychiatric patients.

Similar to any other mathematical model, the proposed model is also based on certain assumptions and approximations that can be challenged in certain real world situations. However, in future associated research, it will be possible to review these assumptions and make refining adjustments to the model in order to render it more accurately reflective of real world conditions. One example of how this refining could be realized would be to experiment with the model by introducing interactions between different cognitive schemas.

\section{CONCLUSION}

This paper has presented a model, which attempts to describe the process of psychopathology development leading to psychiatric illnesses. The theoretical underpinning of the model is based on the general understanding of genetic and environmental interactions and the Cognitive Schema Theory, which explains development of different types of psychopathologies in relation to individual cognitive schemas. The proposed model endeavours to describe the observed changes in psychopathology development in genetically vulnerable individuals under different environmental influences. It is expected that this model can be expanded and will serve as a useful tool that can be used to study the process of psychopathology development in psychiatric illnesses. In the absence of enough mathematical approaches to understand psychiatric illnesses, the proposed model may serve as a stepping-stone and an example that may motivate others to undertake further and similar work in psychiatry, in order to better understand the etiological processes of psychiatric illnesses, and develop more effective treatment interventions.

\section{REFERENCES}

[1] W. W. Eaton, S. S. Martins, G. Nestadt, O. J. Bienvenu, D. Clarke, and P. Alexandre, "The burden of mental disorders," Epidemiologic Reviews, vol. 30, pp. 1-14, November 1, 2008.

[2] J. E. Young, J. S. Klosko, and M. E. Weishaar, Schema therapy: A practitioner's guide, Guilford Press, 2003.

[3] L. J. Eaves, "Genotype environment interaction in psychopathology: Fact or artifact?" Twin Research and Human Genetics, vol. 9, pp. 1-8, 2006.
[4] N. M. Å.VrakiÄ, D. M. Å. VrakiÄ, and C. R. Cloninger, "A general quantitative theory of personality development: Fundamentals of a self-organizing psychobiological complex," Development and Psychopathology, vol. 8, pp. 247-272, 1996.

[5] M. T. Tsuang, W. S. Stone, and J. M. Johnston, Gene-Environment interactions in mental disorders: A current view, New York, NY: Routledge/Taylor and Francis Group, US, 2008.

[6] L. J. Eaves, J. L. Silberg, J. M. Meyer, H. H. Maes, E. Simonoff, A. Pickles, M. Rutter, C. A. Reynolds, A. C. Heath, K. R. Truett, M. C. Neale, M. T. Erikson, R. Loeber, and J. K. Hewitt, "Genetics and developmental psychopathology: 2. The main effects of genes and environment on behavioral problems in the virginia twin study of adolescent behavioral development," Journal of Child Psychology and Psychiatry, vol. 38, pp. 965-980, 1997.

[7] K. P. Lesch, "Gene-Environment interaction and the genetics of depression," Journal of Psychiatry and Neuroscience, vol. 29, pp. 174-184, May 2004.

[8] M. Rutter and J. Silberg, "Gene-Environment interplay in relation to emotional and behavioral disturbance," Annual Review of psychology, vol. 53, pp. 463-490, 2002.

[9] A. D. Ogilvie, S. Battersby, G. Fink, A. J. Harmar, G. M. Goodwin, V. J. Bubb, and C. A. D. Smith, "Polymorphism in serotonin transporter gene associated with susceptibility to major depression," The Lancet, vol. 347, pp. 731-733, 1996.

[10] C. R. Cloninger, S. Siqvardsson, M. Bohman, and A. L. V. Knorring, "Predisposition to petty criminality in swedish adoptees: Ii cross-fostering analysis of gene-environment interaction," Archives of General Psychiatry, vol. 39, pp. 1242-1247, 1982.

[11] S. Freud, "The Ego and the Id.," The Standard Edition of the Complete Psychological Works of Sigmund Freud, vol. 19, Translated and edited by S. J. London, Hogarth Press, 1923.

[12] T. H. Ogden, "The concept of internal object relations," International Journal of Psychoanalysis, vol. 64, pp. 227-241, 1983.

[13] H. Kohut, The analysis of the self: A systematic approach to the psychoanalytic treatment of narcissistic personality disorders, University Of Chicago Press, 2009.

[14] J. Bowlby, Attachment and loss, vol. 1, London: Hogarth, 1969.

[15] J. Bowlby, "Attachment and loss," Separation: Anxiety and anger, London: Hogarth, vol. 2, 1973.

[16] J. Bowlby, "Attachment and loss," Loss: Sadness and depression, London: Hogarth, vol. 3,1980.

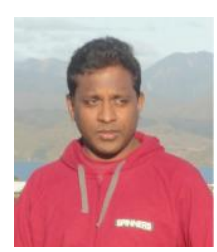

Irosh Fernando was born in Negombo, Sri-Lanka, in 1972. He studied medicine at the University of Colombo, and obtained his MBBS in 2000, and then MD (Psychiatry) in 2006. He also completed the British Computer Society Examination in in 1998, and then obtained an MPhil degree in computer science from the Open University of Colombo in 2008. Whilst he has been working as a medical practitioner since 2001, he also worked as a visiting lecturer in computer science for IDM Negombo andColombo campuses during 1996-2004. He is currentlya Staff Specialist Psychiatrist at Calvary Mater Hopsital, Hunter New England Local Health District, NSW, Australia, and also a PhD candidate in computer science at the School of Electrical Engineering and Computer Science at the University of Newcastle, Australia. Dr Fernando is a fellow of the Royal Australian and New Zealand Collage of Psychatrists(RANZCP), a member of the Institute of Electrical and Electronic Engineers(IEEE), Australian Computer Society (ACS), British Computer Society (BCS), and American Mathematical Society(AMS). 